\title{
Pacific
}

Journal of

Mathematics

\section{AN APPLICATION OF THE VERY WEAK BERNOULLI CONDITION FOR AMENABLE GROUPS}

SCOT Robert AdAms AND JEFFREy EDWARd STEIF 


\title{
AN APPLICATION OF THE VERY WEAK BERNOULLI CONDITION FOR AMENABLE GROUPS
}

\author{
Scot Adams and Jeffrey E. Steif
}

\begin{abstract}
In this paper, we give an application of the recently developed Very Weak Bernoulli condition for amenable groups. The setting for the application is an attractive particle system with the usual lattice replaced by a general countable amenable group.
\end{abstract}

1. Introduction. We consider probability measures on $X=\{0,1\}^{G}$ which are invariant under the natural right action of the group $G$. Two such measures $\mu$ and $\nu$ are isomorphic if there exists an $f:\left(\{0,1\}^{G}, \mu\right) \rightarrow\left(\{0,1\}^{G}, \nu\right)$ which is bijective a.e. and measurepreserving and which commutes with the action of $G$. A Bernoulli Shift is a stationary process which is isomorphic to an i.i.d. process. For the case $G=Z$, it was proven by Ornstein [6] that entropy is a complete invariant for Bernoulli Shifts. In this work, a number of important properties of a finite state discrete time $(G=Z)$ stationary process were introđuced, namely Finitely Determined (FD), Very Weak Bernoulli (VWB), and Weak Bernoulli (WB). This work together with [10] shows that FD and VWB are equivalent to being a Bernoulli Shift. While these are also all implied by the WB condition, they do not imply it. The fact that a Bernoulli Shift is not necessarily WB might seem strange but is partially explained by the fact that WB is not an isomorphism invariant. An example of such a process is given in [13]. The equivalence of Bernoulli and VWB allowed researchers to prove that a number of concrete systems were in fact Bernoulli Shifts.

After this, further equivalent but useful concepts were introduced, namely that of Thouvenot's extremality [11] and that of $\varepsilon$-block independence [12]. Later on, it was natural to extend as much as possible the Bernoulli theory to the group $Z^{d}$ and ultimately to amenable groups. Kieffer [4] succeeded in obtaining a Shannon-McMillan theorem in the amenable group setting. (This was later improved to a pointwise theorem for certain Følner sequences in [8].) Recently, the theorem that entropy is a complete invariant for Bernoulli Shifts was generalized to the case where the group acting is a general countable, discrete, amenable group [7]. (In fact, they handle the broader class 
of unimodular amenable groups "with a good entropy theory"; this includes most naturally occurring unimodular amenable groups.)

It is fairly straightforward to extend the definition of VWB to the group $Z^{d}$. In [3], analogues of extremality and $\varepsilon$-block independence for $Z^{d}$ are used. The concept of extremality for an amenable group is also used in [7]. The analogue of FD to $Z^{d}$ (and to amenable groups) is obvious. However, it was not at all clear what the analogue of the above VWB condition should be in this more general context of an amenable group.

In [1], a natural notion called Følner Independence (FI) is introduced. This condition makes sense for a general countable amenable group and implies that the system is a Bernoulli Shift. In [1], it is shown that the ferromagnetic Ising model on an amenable group in either its plus or minus states are FI and hence are Bernoulli Shifts. (This generalizes a result in [Ornstein and Weiss, unpublished].) However, even for the simple case of the group $Z$, FI is strictly stronger than Bernoulli (see [9]).

In another recent paper [2], the notion of VWB for the group $Z^{d}$ is extended to a general countable amenable group. This condition, although much more complicated than FI, is equivalent to Bernoulli and moreover is similar in spirit to the VWB condition for $Z^{d}$. In this work is the idea of ordering a series of blocks inside a larger Følner block.

The purpose of this paper is to give an application of this new VWB condition for amenable groups. We show that a large collection of Markov processes (whose state space is the collection of 0,1 configurations on a countable, discrete, amenable group) are Bernoulli Shifts. These results generalize the results in [14] to the amenable group context providing at the same time an application of the new VWB condition for amenable groups. In order to do this, we introduce a condition called Time Følner Independence (Definition 2.5.1) which is stronger than VWB, but weaker than Følner Independence. This condition makes sense only for amenable groups with a distinguished time direction (i.e., for groups of the form $G \times Z$ where $G$ is amenable).

An example of a discrete amenable group is the integer Heisenberg group: the group of all $3 \times 3$, upper triangular, unipotent matrices (with integer entries). This group is the same as $Z^{3}$ with multiplication twisted by the Heisenberg cocycle: $(a, b, c) \cdot(x, y, z)=$ $(a+x, b+y, c+a y+z)$. For this group, the Interacting Particle 
Systems defined below can still be thought of as being defined on the usual lattice $Z^{3}$. However, the usual additive translation invariance is replaced by some more complicated notion of invariance.

We now introduce the specific processes which we will analyze. We will consider Probabilistic Cellular Automata (PCA). These will be discrete time Markov processes with state space $X=\{0,1\}^{G}$, the set of configurations of 0 's and 1's on a countable amenable group $G$. The transitions will be governed by a family of functions

$$
\{c(x, \eta)\}_{x \in G, \eta \in X}
$$

where

$$
B=\sup _{x \in G, \eta \in X} c(x, \eta) \leq 1 / 2
$$

and $c(x, \eta)$ is, for fixed $x$, a continuous function of $\eta$ where $X$ is given the product topology. The reason for having the $1 / 2$ bound rather than the more natural bound of 1 is explained later.

The evolution of our process is defined as follows. If the state of the system is $\eta$, then at the next stage each lattice point $x$ in $G$ switches its value independently with probability $c(x, \eta)$. In particular, this yields a product measure at the next stage, which we denote by $T \eta$. We can then evolve any initial distribution $\nu$, and we denote this evolved measure by $T \nu$ where $T \nu=\int_{X} T \eta d \nu(\eta)$. The measure $\mu$ is called stationary if $T \mu=\mu$.

We note that $X$ has a natural partial order defined on it: $\eta \preceq \delta$ if $\eta(x) \leq \delta(x)$ for all $x \in G$.

Definition 1.1. A PCA (IPS) is attractive if, whenever $\eta \preceq \delta$, then $c(x, \eta) \leq c(x, \delta)$ if $\eta(x)=\delta(x)=0$ and $c(x, \eta) \geq c(x, \delta)$ if $\eta(x)=\delta(x)=1$.

Heuristically, one has that 0's attract 0's and 1's attract 1's. In this paper, we deal exclusively with attractive systems.

There is another characterization of attractiveness which will be useful for us later. We say that a function $f$ from $X$ to $R$ is increasing if $\eta \preceq \delta$ implies that $f(\eta) \leq f(\delta)$. Let $\mathscr{M}$ denote the collection of increasing continuous functions on $X$. This then allows us to place a natural partial order on $P(X)$. in $\mathscr{M}$.

Definition 1.2. $\nu \preceq \mu$ if $\int_{X} f d \nu \leq \int_{X} f d \mu$ for all functions $f$

The fact that $\preceq$ is a partial order on $P(X)$ is easily verified, as is the continuity of $\preceq$ with respect to the weak topology in that $\nu_{n} \preceq \mu_{n}$ for all $n, \nu_{n} \rightarrow \nu$ and $\mu_{n} \rightarrow \mu$ imply that $\nu \preceq \mu$. 
We now present an alternative definition of attractiveness in the following proposition (see [5]).

Proposition 1.3. A PCA (IPS) is attractive if and only if $\nu \preceq \mu$ implies $T \nu \preceq T \mu$.

In the attractive context, there are two distinguished stationary distributions which might reduce to the same one. The next proposition deals with one of these.

Proposition 1.4. $\lim _{n \rightarrow \infty} T^{n} \delta_{0}$ exists and is a stationary distribution where $\delta_{0}$ denotes the unit point mass at the configuration of all 0's. Furthermore, this limiting distribution is smaller than any other stationary distribution with respect to the partial order defined in Definition 1.2.

The analogous result holds when 0 is replaced by 1 . For the proof, see [5]. We denote by $\underline{\nu}$ the stationary distribution whose existence is guaranteed by the above proposition. Similarly, if we start with the configuration of all 1's, we denote the limiting stationary distribution by $\bar{\nu}$. The reason why we take $1 / 2$ as a uniform bound on the spin rates is that Proposition 1.3 and 1.4 become false otherwise, which one can see by taking $c(x, \eta) \equiv 1$.

We only consider spin rates which are translation invariant in an appropriate sense. We first note that $G$ acts canonically on itself by right translations. Next, $G$ acts canonically on $X$ on the right by $(\eta g)(x)=\eta\left(x g^{-1}\right)$. Finally, $G$ acts on the collection of spin rates on the right by $c(x, \eta) g=c(x g, \eta g)$. We only consider spin rates which are fixed under this action, i.e., are (right) translation invariant.

Letting $\underline{\tilde{\nu}}$ be the stationary process with stationary distribution $\underline{\nu}$, we have that $\underline{\tilde{\nu}}$ is a process indexed by $G \times Z$ which is (right) translation invariant whose proof we leave to the reader. Our main theorem is

\section{THEOREM 1.5. $\underline{\tilde{\nu}}$ is a Bernoulli Shift.}

In $\S 2$ we develop the notion of Time Følner Independence and prove that it implies VWB (Theorem 2.5.2). In $\S 3$, we prove Theorem 1.5 using Theorem 2.5.2.

2. Time Følner Independence implies VWB. In this section we prove (Theorem 2.5.2) that Time Følner Independence implies Very Weak 
Bernoulli, which is equivalent to Bernoulli [2, Theorem 3.7 and 7, 1987, Theorem 8, p. 93].

2.1. Preliminaries. We recall the $\bar{d}$-metric: Let $X=\left\{X^{i}\right\}_{i \in I}$ and $Y=\left\{Y^{i}\right\}_{i \in I}$ be finite processes (i.e., processes with $|I|<\infty$ ) and let $m$ be any coupling of $X$ with $Y$. We define

$$
\bar{d}^{m}(X, Y):=\frac{1}{|I|} E^{m}\left[\left|\left\{i \in I \mid X^{i} \neq Y^{i}\right\}\right|\right] .
$$

We define $\bar{d}(X, Y):=\inf _{m} \bar{d}^{m}(X, Y)$, where the infimum is taken over all couplings $m$ of $X$ and $Y$.

Recall [1, Definition 2.8] that if $X$ and $Y$ are coupled finite processes, then $Y$ is $\varepsilon$-process independent of $X$ if $\bar{d}_{X}(X \vee Y, X \| Y)<\varepsilon$. The next lemma allows us to reformulate this definition using the ordinary $\bar{d}$-metric instead of the $\bar{d}_{X}$-metric of [1, Definition 2.4].

Lemma 2.1.1. Let $X$ and $Y$ be coupled finite processes. Let $\mathscr{C}(X)$ denote the configurations of $X$ of positive probability. For every $x \in$ $\mathscr{C}(X)$, let $Y_{x}$ denote the process $Y$, conditioned on $X=x$.

(i) If $Y$ is $\varepsilon$-process independent of $X$, then there exists $C \subseteq$ $\mathscr{C}(X)$ such that

$$
\operatorname{Pr}[X \in C]>1-\sqrt{\varepsilon}
$$

and such that, for all $x \in C, \bar{d}\left(Y_{x}, Y\right)<\sqrt{\varepsilon}$.

(ii) Assume there exists $C \subseteq \mathscr{C}(X)$ satisfying

$$
\operatorname{Pr}[X \in C]>1-\varepsilon
$$

and $\bar{d}\left(Y_{x}, Y\right)<\varepsilon$, for all $x \in C$. Then $Y$ is $[\varepsilon+(1-\varepsilon) \varepsilon]$-process independent of $X$.

Proof. By [1, Lemma 2.6],

$$
\bar{d}_{X}(X \vee Y, X \| Y)=\sum_{x \in \mathscr{C}(X)} \operatorname{Pr}[X=x] \cdot \bar{d}\left(Y_{x}, Y\right) .
$$

From this, the lemma is easily obtained.

Recall [1, §3] that, if $F \subseteq G$ is finite and if $\delta>0$, then a finite subset $K \subseteq G$ is $(F, \delta)$-invariant if, for all $f \in F,|f K \Delta K|<\delta|K|$. We say that a property of finite subsets of $G$ holds "for all sufficiently invariant subsets" if: there exists $(F, \delta)$ such that it holds for all $(F, \delta)$-invariant subsets. Recall also that $\partial_{F} K$ denotes the set of all $a \in G$ such that $F a \cap K \neq \varnothing \neq F a \cap(G \backslash K)$. 
The group $G$ is said to be amenable if, for all finite $F \subseteq G$, for all $\delta>0$, there exists a $(F, \delta)$-invariant (finite) subset of $G$.

Definition 2.1.2 [1, Definition 3.3]. Let $\varepsilon>0$. A collection $A_{1}, \ldots, A_{k}$ of finite subsets of $G$ is said to be a disjoint $\varepsilon$-quasitiling system if, for all sufficiently invariant sets $F$, there exist disjoint subsets $\bar{A}_{1}, \ldots, \bar{A}_{m} \subseteq F$ such that

(1) each $\bar{A}_{p}$ is a right translate of some $A_{j}$; and

(2) $\left|F \backslash \bigcup_{p} \bar{A}_{p}\right|<\varepsilon|F|$.

If such sets exist, then we say that $F$ is disjoint $\varepsilon$-quasi-tilable by $A_{1}, \ldots, A_{k}$ and we say that $\bar{A}_{1}, \ldots, \bar{A}_{m}$ is a disjoint $\varepsilon$-quasi-tiling of $F$ by right translates of $A_{1}, \ldots, A_{k}$.

Definition 2.1.3 [2, Definition 3.1]. We say that a stationary $G$ process $X$ is Very Weak Bernoulli if, for all $\varepsilon>0$, there exists a disjoint $\varepsilon$-quasi-tiling system $A_{1}, \ldots, A_{k}$, such that for all sufficiently invariant finite sets $K \subseteq G$, there exists an ordered disjoint $\varepsilon$-quasitiling $\bar{A}_{1}, \ldots, \bar{A}_{m}$ of $K$ such that for all $p=1, \ldots, m$, the process $X_{\bar{A}_{p}}$ is $\varepsilon$-process independent of $X_{\bar{P}_{p}}$, where $\bar{P}_{p}:=\bar{A}_{1} \cup \cdots \cup \bar{A}_{p-1}$. An ordered disjoint $\varepsilon$-quasi-tiling with this property will be said to be $\varepsilon$-almost independent under $X$.

Let $G$ be a countable, discrete, amenable group with identity element $e$.

Let $K \subseteq G \times Z$ be finite and nonempty. For $-\infty<a<b<\infty$, define

$$
\begin{aligned}
Z_{a}^{b} & :=Z \cap[a, b], \\
Z_{-\infty}^{b} & :=Z \cap(-\infty, b], \\
Z_{a}^{\infty} & :=Z \cap[a, \infty), \\
K_{a}^{b} & :=K \cap\left(G \times Z_{a}^{b}\right), \\
K_{a} & :=\{g \in G \mid(g, a) \in K\}, \\
\operatorname{bot}(K) & :=\min \left\{a \in Z \mid K_{a} \neq \varnothing\right\}, \\
\operatorname{top}(K) & :=\max \left\{a \in Z \mid K_{a} \neq \varnothing\right\}, \\
\operatorname{intvl}(K) & :=Z_{\operatorname{bot}(K)}^{\operatorname{top}(K)} .
\end{aligned}
$$

For $N \in Z_{1}^{\infty}$, define

$$
\operatorname{Slice}_{N}(K):=\{t \in \operatorname{intvl}(K) \mid[t-\operatorname{bot}(K)] \in N Z\} .
$$

For $N \in Z_{1}^{\infty}$ and $S \subseteq \operatorname{Slice}_{N}(K)$, define

$$
\operatorname{Size}_{N}^{K}(S):=\sum_{s \in S}\left|K_{s}^{s+N-1}\right|,
$$

where $|\cdot|$ denotes cardinality. 
If $A$ and $B$ are sets, then we define $A \triangle B:=(A \backslash B) \cup(B \backslash A)$.

2.2. Cylindrical sets. Let $G$ be a countable, discrete, amenable group with identity element $e$.

Definition 2.2.1. Let $L \subseteq G \times Z$ be finite and nonempty. We say that $L$ is cylindrical if $L_{i}=L_{j}$, for all $i, j \in \operatorname{intvl}(L)$. If $\zeta>0$, then we say that $L$ is $\zeta$-cylindrical if $\left|L_{i} \Delta L_{j}\right|<\zeta\left|L_{i}\right|$, for all $i, j \in$ $\operatorname{intvl}(L)$.

LemMA 2.2.2. Let $\zeta>0$ and let $N \in Z_{1}^{\infty}$ be given. Then, for all sufficiently invariant $K \subseteq G \times Z: K$ is $(\zeta, N)$-slice-cylindrical, i.e., there exists $S \subseteq$ Slice $_{N}(K)$ such that

(i) $\operatorname{Size}_{N}^{K}(S)>(1-\zeta)|K|$; and

(ii) for all $s \in S, K_{s}^{s+N-1}$ is $\zeta$-cylindrical.

Proof. We may assume that $\zeta<2$. Choose $\delta>0$ such that $4 \sqrt{2 N \delta}<\zeta$. Let $K \subseteq G \times Z$ be $\left(\{e\} \times Z_{1-N}^{N-1}, \delta\right)$-invariant. We will show that $K$ is $(\zeta, N)$-slice-cylindrical.

Let

$$
B:=\bigcup_{t \in Z_{1-N}^{N-1}}\{[(e, t) K] \triangle K\} .
$$

Then $|B| \leq(2 N-1) \delta|K|<2 N \delta|K|$. Let

$$
S:=\left\{s \in \operatorname{Slice}_{N}(K)|| B_{s}^{s+N-1}|<\sqrt{2 N \delta}| K_{s}^{s+N-1} \mid\right\} .
$$

Then

$$
\operatorname{Size}_{N}^{K}(S)>(1-\sqrt{2 N \delta})|K|>[1-(\zeta / 4)]|K|>(1-\zeta)|K|,
$$

verifying (i).

Fix $s \in S$. Fix $i, j \in Z_{s}^{s+N-1}$. To prove (ii), it suffices to show that $\left|K_{i} \triangle K_{j}\right|<\zeta\left|K_{i}\right|$.

Since

$$
\left|B_{s}^{s+N-1}\right|<\sqrt{2 N \delta}\left|K_{s}^{s+N-1}\right|<\zeta\left|K_{s}^{s+N-1}\right| / 4,
$$

it follows that there exists $k_{0} \in Z_{s}^{s+N-1}$ such that $\left|B_{k_{0}}\right|<\zeta\left|K_{k_{0}}\right| / 4$.

Define

$$
B^{\prime}:=K \backslash\left[\left(e, k_{0}-i\right) K\right] \text { and } B^{\prime \prime}:=\left[\left(e, k_{0}-i\right) K\right] \backslash K .
$$

Then $B^{\prime} \subseteq B, B^{\prime \prime} \subseteq B, K_{k_{0}} \backslash K_{i} \subseteq B_{k_{0}}^{\prime}$ and $K_{i} \backslash K_{k_{0}} \subseteq B_{k_{0}}^{\prime \prime}$. Therefore $K_{i} \triangle K_{k_{0}} \subseteq B_{k_{0}}^{\prime} \cup B_{k_{0}}^{\prime \prime} \subseteq B_{k_{0}}$. Similarly, $K_{j} \triangle K_{k_{0}} \subseteq B_{k_{0}}$. 
Then $\left|K_{i} \triangle K_{k_{0}}\right| \leq\left|B_{k_{0}}\right|<\zeta\left|K_{k_{0}}\right| / 4$. Similarly, $\left|K_{j} \triangle K_{k_{0}}\right| \leq\left|B_{k_{0}}\right|<$ $\zeta\left|K_{k_{0}}\right| / 4$.

Now $\left|K_{k_{0}}\right| \leq\left|K_{i}\right|+\left|K_{k_{0}} \backslash K_{i}\right|$ and $K_{k_{0}} \backslash K_{i} \subseteq K_{i} \Delta K_{k_{0}}$, so

$$
\left|K_{i}\right| \geq\left|K_{k_{0}}\right|-\left(\zeta\left|K_{k_{0}}\right| / 4\right)>\left|K_{k_{0}}\right| / 2,
$$

since $\zeta<2$. Then

$$
\begin{aligned}
\left|K_{i} \Delta K_{j}\right| & \leq\left|K_{i} \Delta K_{k_{0}}\right|+\left|K_{k_{0}} \Delta K_{j}\right| \\
& <\left(\zeta\left|K_{k_{0}}\right| / 4\right)+\left(\zeta\left|K_{k_{0}}\right| / 4\right)=\zeta\left|K_{k_{0}}\right| / 2<\zeta\left|K_{i}\right|
\end{aligned}
$$

2.3. Localization of invariance. Let $G$ be a countable, discrete, amenable group with identity element $e$.

Lemma 2.3.1. Let $F \subseteq G$ be finite, let $\eta>0$ and let $N \in Z_{1}^{\infty}$. Then, for all sufficiently invariant $K \subseteq G \times Z, K$ is $(F, \eta, N)$-slice invariant, i.e., there exists $S \subseteq \operatorname{Slice}_{N}(K)$ such that

(i) $\operatorname{Size}_{N}^{K}(S)>(1-\eta)|K|$; and

(ii) for all $s \in S$, there exists $i \in Z_{s}^{s+N-1}$ such that $K_{i}$ is $(F, \eta)$ invariant.

Proof. Let $K$ be $\left(F \times\{0\}, \eta^{2} /|F|^{2}\right)$-invariant. We will show that $K$ is $(F, \eta, N)$-slice-invariant. Let $S$ consist of all $s \in \operatorname{Slice}_{N}(K)$ such that $K_{s}^{s+N-1}$ is $(F \times\{0\}, \eta /|F|)$-invariant. We wish to prove (i) and (ii) of Lemma 2.3.1.

We first prove (i). Assume $\operatorname{Size}_{N}^{K}(S) \leq(1-\eta)|K|$. We aim for a contradiction. Let $\widetilde{S}:=\operatorname{Slice}_{N}(K) \backslash S$ so that $\operatorname{Size}_{N}^{K}(\widetilde{S}) \geq \eta|K|$. Then, for all $s \in \widetilde{S}$, there exists $f_{s} \in F$ such that

$$
\left|\left(f_{s}, 0\right) K_{s}^{s+N-1} \triangle K_{s}^{s+N-1}\right| \geq \eta\left|K_{s}^{s+N-1}\right| /|F| .
$$

For all $f \in F$, let $\widetilde{S}(f):=\left\{s \in \widetilde{S} \mid f_{s}=f\right\}$. As $\operatorname{Size}_{N}^{K}(\widetilde{S}) \geq \eta|K|$, it follows that, for some $f_{0} \in F$,

$$
\operatorname{Size}_{N}^{K}\left(\widetilde{S}\left(f_{0}\right)\right) \geq \eta|K| /|F| .
$$

Let

$$
\widetilde{K}:=\bigcup_{s \in \widetilde{S}\left(f_{0}\right)} K_{s}^{s+N-1} .
$$

Then $|\widetilde{K}|=\operatorname{Size}_{N}^{K}\left(\widetilde{S}\left(f_{0}\right)\right) \geq \eta|K| /|F|$, so, by definition of $\widetilde{S}\left(f_{0}\right)$,

$$
\left|\left(f_{0}, 0\right) \widetilde{K} \triangle \widetilde{K}\right| \geq \eta|\widetilde{K}| /|F| \geq \eta^{2}|K| /|F|^{2} \text {. }
$$


Since $\widetilde{K}$ is a union of sets of the form $K_{i} \times\{i\}$, it follows that

$$
\left(f_{0}, 0\right) \widetilde{K} \Delta \widetilde{K} \subseteq\left(f_{0}, 0\right) K \triangle K,
$$

and hence $\left|\left(f_{0}, 0\right) K \Delta K\right| \geq \eta^{2}|K| /|F|^{2}$. But $K$ is $\left(F \times\{0\}, \eta^{2} /|F|^{2}\right)$ invariant, a contradiction.

We now prove (ii). Fix $s \in S$ and assume, for all $i \in Z_{s}^{s+N-1}$, that $K_{i}$ is not $(F, \eta)$-invariant, i.e., there exists $f_{i} \in F$ such that

$$
\left|f_{i} K_{i} \Delta K_{i}\right| \geq \eta\left|K_{i}\right|
$$

We aim for a contradiction.

Let $L:=K_{s}^{s+N-1}$. For $f \in F$, let $I(f):=\left\{i \in Z_{s}^{s+N-1} \mid f_{i}=f\right\}$. Then there exists $f_{1} \in F$ such that

$$
\sum_{i \in I\left(f_{1}\right)}\left|K_{i}\right| \geq|L| /|F| .
$$

Let $\bar{L}:=\bigcup_{i \in I\left(f_{1}\right)} L_{i} \times\{i\}$. Then

$$
\left|\left(f_{1}, 0\right) \bar{L} \Delta \bar{L}\right| \geq \eta|\bar{L}| \geq \eta|L| /|F| \text {. }
$$

So, since $\bar{L}$ is a union of sets of the form $L_{i} \times\{i\}$, it follows that

$$
\left(f_{1}, 0\right) \bar{L} \Delta \bar{L} \subseteq\left(f_{1}, 0\right) L \triangle L,
$$

and hence $\left|\left(f_{1}, 0\right) L \Delta L\right| \geq \eta|L| /|F|$. However, since $s \in S, L=$ $K_{s}^{s+N-1}$ is $(F \times\{0\}, \eta /|F|)$-invariant, so $\left|\left(f_{1}, 0\right) L \triangle L\right|<\eta|L| /|F|$, a contradiction.

2.4. Prolongation of tilings. Let $G$ be a countable, discrete, amenable group with identity element $e$.

By [1, Lemma 3.4], there exist disjoint $\varepsilon$-quasi-tiling systems (Definition 2.1.2) consisting of arbitrarily invariant subsets of $G$.

If $\mathscr{A}$ is a collection of subsets of $G$ and if $I \subseteq Z$, then we define

$$
\mathscr{A} \times I:=\{A \times I \mid A \in \mathscr{A}\} .
$$

We call $\mathscr{A} \times I$ the I-prolongation of $\mathscr{A}$.

LemmA 2.4.1. Let $N \in Z_{1}^{\infty}$ and $\lambda>0$. Then there exists $\zeta>0$ such that

if $\mathscr{A}$ is a disjoint $\zeta$-quasi-tiling system for $G$;

if $L \subseteq G \times Z$ is finite and $\zeta$-cylindrical;

if $|\operatorname{intvl}(L)| \leq N$; and

if, for some $i_{0} \in \operatorname{intvl}(L), L_{i_{0}}$ is disjoint $\zeta$-quasi-tilable by $\mathscr{A}$, then $L$ is disjoint $\lambda$-quasi-tilable by $\mathscr{A} \times \operatorname{intvl}(L)$. 
Proof. Choose $\zeta>0$ such that

$$
(2+N) \zeta(1+\zeta)<\lambda
$$

Let $I:=\operatorname{intvl}(L)$. Then $|I| \leq N$ by hypothesis. Let $\overline{\mathscr{A}}$ be a disjoint $\zeta$-quasi-tiling of $L_{i_{0}}$ by right translates of elements of $\mathscr{A}$. Let

$$
\widetilde{\mathscr{A}}:=\{\bar{A} \in \overline{\mathscr{A}} \mid \bar{A} \times I \subseteq L\} \text {. }
$$

We wish to show that

$$
|L \backslash \bigcup(\widetilde{\mathscr{A}} \times I)|<\lambda|L|
$$

Fix $j \in I$. It suffices to show that

$$
\left|L_{j} \backslash \bigcup \widetilde{\mathscr{A}}\right|<\lambda\left|L_{j}\right|
$$

By definition of $\widetilde{A}$,

$$
\bigcup(\overline{\mathscr{A}} \backslash \widetilde{\mathscr{A}}) \subseteq\left\{g \in \bigcup \overline{\mathscr{A}} \mid g \notin L_{k} \text {, for some } k \in I\right\} .
$$

But $\bigcup \overline{\mathscr{A}} \subseteq L_{i_{0}}$, so

$$
\bigcup(\overline{\mathscr{A}} \backslash \widetilde{\mathscr{A}}) \subseteq \bigcup_{k \in I} L_{i_{0}} \Delta L_{k}
$$

Since $L$ is $\zeta$-cylindrical, it follows that $|\bigcup(\overline{\mathscr{A}} \backslash \widetilde{\mathscr{A}})| \leq|I| \zeta\left|L_{i_{0}}\right| \leq$ $N \zeta\left|L_{i_{0}}\right|$. Thus

$$
\left|L_{i_{0}} \backslash \bigcup \widetilde{\mathscr{A}}\right|=\left|L_{i_{0}} \backslash \bigcup \overline{\mathscr{A}}\right|+|\bigcup(\overline{\mathscr{A}} \backslash \widetilde{\mathscr{A}})|<\zeta\left|L_{i_{0}}\right|+N \zeta\left|L_{i_{0}}\right| .
$$

Since $L$ is $\zeta$-cylindrical, it follows that $\left|L_{j} \backslash L_{i_{0}}\right| \leq\left|L_{i_{0}} \triangle L_{j}\right|<\zeta\left|L_{i_{0}}\right|$, so

$$
\left|L_{j} \backslash \bigcup \widetilde{\mathscr{A}}\right| \leq\left|L_{j} \backslash L_{i_{0}}\right|+\left|L_{i_{0}} \backslash \bigcup \widetilde{\mathscr{A}}\right|<(2+N) \zeta\left|L_{i_{0}}\right|
$$

Using $\zeta$-cylindricity of $L$ once more,

$$
\left|L_{i_{0}}\right| \leq\left|L_{j}\right|+\left|L_{i_{0}} \backslash L_{j}\right| \leq\left|L_{j}\right|+\left|L_{i_{0}} \Delta L_{j}\right|<(1+\zeta)\left|L_{j}\right| .
$$

Thus

$$
\left|L_{j} \backslash \bigcup \widetilde{\mathscr{A}}\right|<(2+N) \zeta(1+\zeta)\left|L_{j}\right|<\lambda\left|L_{j}\right|
$$

2.5. Time Følner independence. Let $G$ be a countable, discrete, amenable group with identity element $e$. Let $X$ be a right stationary $(G \times Z)$-process.

Definition 2.5.1. We say that $X$ is Time Følner Independent if, for all $\varepsilon>0$, there exists $N \in Z_{1}^{\infty}$ such that, for any sufficiently 
invariant $A \subseteq G$ :

$A$ is $(N, \varepsilon)$-wide, i.e., for all finite

$$
S \subseteq\left(G \times Z_{-\infty}^{N}\right) \backslash\left(A \times Z_{1}^{N}\right),
$$

the process $X \mid\left(A \times Z_{1}^{N}\right)$ is $\varepsilon$-process independent of $X \mid S$.

Recall (Definition 2.1.3) the definition of Very Weak Bernoulli. We now come to the main result of this section:

THEOREM 2.5.2. If $X$ is Time Følner Independent, then $X$ is Very Weak Bernoulli.

Proof. Let $\varepsilon>0$ be given. We wish to find a disjoint $\varepsilon$-quasi-tiling system satisfying the condition of Definition 2.1.3.

Choose $N$ as in Definition 2.5.1. Let $\lambda:=\varepsilon / 3$. Choose $\zeta$ as in Lemma 2.4.1. We may assume that $\zeta<\varepsilon / 3$.

By [1, Lemma 3.4], we may choose in $G$ a disjoint $\zeta$-quasi-tiling system $\mathscr{A}$ such that every $A \in \mathscr{A}$ is $(N, \varepsilon)$-wide (Definition 2.5.1). We will verify that $\mathscr{A} \times Z_{1}^{N}$ is a disjoint $\varepsilon$-quasi-tiling system, satisfying the requirements of Definition 2.1.3.

Choose a finite subset $F \subseteq G$ and $\eta>0$ such that any $(F, \eta)$ invariant subset of $G$ is disjoint $\zeta$-quasi-tilable by right translates of elements of $\mathscr{A}$. We may assume that $\eta<\varepsilon / 3$.

By Lemmas 2.2.2 and 2.3.1, it suffices to show that if $K \subseteq G \times Z$ is $(\zeta, N)$-slice-cylindrical and $(F, \eta, N)$-slice-invariant, then $K$ admits an $\varepsilon$-almost independent ordered $\varepsilon$-quasi-tiling (by right translates of elements of $\left.\mathscr{A} \times Z_{1}^{N}\right)$. So fix such a $K$.

Then, by Lemmas 2.2.2 and 2.3.1, there exists $S \subseteq \operatorname{Slice}_{N}(K)$ such that

(i) $\operatorname{Size}_{N}^{K}(S)>(1-\zeta-\eta)|K|$;

(ii) for all $s \in S, K_{s}^{s+N-1}$ is $\zeta$-cylindrical; and

(iii) for all $s \in S$, there exists $i \in Z_{s}^{s+N-1}$ such that $K_{i}$ is $(F, \eta)$ invariant.

Fix, for a moment, some $s \in S$. Let $L:=K_{s}^{s+N-1}$. Then there exists $i \in Z_{s}^{s+N-1}=\operatorname{intvl}(L)$ such that $L_{i}$ is $(F, \eta)$-invariant. By the definition of $F$ and $\eta, L_{i}$ is disjoint $\zeta$-quasi-tilable by right translates of elements of $\mathscr{A}$. On the other hand, by (ii), $L=K_{s}^{s+N-1}$ is $\zeta$-cylindrical. We apply Lemma 2.4 .1 and conclude that $L$ is disjoint $\lambda$-quasi-tilable by right translates of elements of $\mathscr{A} \times \operatorname{intvl}(L)=$ $\mathscr{A} \times Z_{s}^{s+N-1}$. Let $\overline{\mathscr{A}}_{s}$ be such a disjoint $\lambda$-quasi-tiling. That is, 
defining $C_{s}:=\bigcup_{\bar{A} \in \overline{\mathscr{A}}_{s}} \bar{A}$, we have:

(a) every element of $\overline{\mathscr{A}}_{s}$ is a right translate of some element of $\mathscr{A} \times Z_{s}^{s+N-1}$ (and is therefore a right translate of some element of $\left.\mathscr{A} \times Z_{1}^{N}\right)$

(b) the elements of $\overline{\mathscr{A}}_{s}$ are pairwise disjoint;

(c) $C_{s} \subseteq K_{s}^{s+N-1}$; and

(d) $\left|C_{s}\right|>(1-\lambda)\left|K_{s}^{s+N-1}\right|$.

Now choose any ordering of the elements of

$$
\overline{\mathscr{A}}:=\bigcup_{s \in S} \overline{\mathscr{A}}_{s}
$$

such that if $s<s^{\prime}$, then the elements of $\overline{\mathscr{A}}_{s}$ precede the elements of $\overline{\mathscr{A}}_{s^{\prime}}$.

Let $\widetilde{S}:=\operatorname{Slice}_{N}(K) \backslash S$. By (i) above, $\operatorname{Size}_{N}^{K}(\widetilde{S})<(\zeta+\eta)|K|$. Then

$$
\begin{aligned}
|K \backslash \bigcup \overline{\mathscr{A}}| & =\left|\bigcup_{s \in \widetilde{S}} K_{s}^{s+N-1}\right|+\left|\bigcup_{s \in S}\left(K_{s}^{s+N-1} \backslash C_{S}\right)\right| \\
& <(\zeta+\eta)|K|+\sum_{s \in S} \lambda\left|K_{s}^{s+N-1}\right| .
\end{aligned}
$$

Then, since $\zeta<\varepsilon / 3, \eta<\varepsilon / 3$ and $\lambda=\varepsilon / 3$, we obtain $|K \backslash \bigcup \overline{\mathscr{A}}|<$ $\varepsilon|K|$.

The elements of $\overline{\mathscr{A}}$ are pairwise disjoint and contained in $K$.

Now, $X$ is right stationary and each $A \in \mathscr{A}$ is $(N, \varepsilon)$-wide. Therefore, the Time Følner Independence condition (Definition 2.5.1) implies that $\overline{\mathscr{A}}$ (with the chosen ordering) is $\varepsilon$-almost independent, as required by Definition 2.1.3.

3. $\underline{\tilde{v}}$ is Bernoulli. In this section, we prove Theorem 1.5. Most of the proof follows that in [14], where the group $G$ is $Z^{d}$ for some $d$. We in fact sometimes simply refer to the proofs in this paper explaining the necessary modifications. Let $\left\{Y_{i}\right\}_{i \in Z}$ denote the stationary process with stationary distribution $\underline{\nu}$. So $Y_{i}(x)$ is the value of the process at time $i$ and location $x$. Let $\left\{Y_{i}^{\eta}\right\}_{i \geq 0}$ denote the process $\left\{Y_{i}\right\}_{i \geq 0}$ conditioned on $\eta$ at time 0 .

Let $G$ be a countable, discrete, amenable group with identity ele ment $e$.

LEMMA 3.1. If the spin rates are attractive, then given $\varepsilon>0$, there is an $N$ and a set $A \subseteq X$ of $\underline{\nu}$-measure at least $1-\varepsilon$ so that for all 
$n \geq N$ and for all $\eta \in A$,

$$
\bar{d}\left(\left\{Y_{i}(e)\right\}_{i=1}^{n},\left\{Y_{i}^{\eta}(e)\right\}_{i=1}^{n}\right) \leq \varepsilon .
$$

Proof. This is proved exactly as is Lemma 4.2 in [14].

To prove Theorem 1.5, we first deal with finite range spin rates (defined below) and then extend to infinite range spin rates. We prove this by showing that $\underline{\tilde{\nu}}$ has the Time Følner Independent property and then invoking Theorem 2.5.2.

Proposition 3.2. Given $\varepsilon>0$, there is an integer $b$ such that for all sufficiently invariant $F \subseteq G$, there is a set $E \subseteq X$ (depending on $F)$ of $\underline{\nu}$-measure at most $\varepsilon$ such that

$$
\bar{d}\left(\left\{Y_{i}^{\eta}(x)\right\}_{(x, i) \in F \times[1, b]},\left\{Y_{i}(x)\right\}_{(x, i) \in F \times[1, b]} \leq 8 \varepsilon^{1 / 4}\right.
$$

for all $\eta \notin E$.

Proof. This is proved exactly as is Proposition 4.3 in [14]. There are three minor changes one must make. The place where the pointwise ergodic theorem is used is replaced by the mean ergodic theorem which is really all that is needed. (It is not known if every amenable group admits a Følner sequence for which the pointwise ergodic theorem holds. However, the mean ergodic theorem is valid. This also explains why the statement here is slightly weaker than that of Proposition 4.3.) One must also show that a $\bar{d}$-limit of mixing measures is mixing and that a direct product of two mixing measures is ergodic. However, these facts are proved in the same way as they are for $Z$-actions.

The next lemma shows that for finite range systems, the process conditioned on $\eta$ at time 0 in $F \times[1, b]$ is $\bar{d}$-close to itself if we further condition on elements in $G \times Z$ whose spatial coordinates do not lie in $F$ and whose time point is in $[1, b]$ providing $F$ is sufficiently invariant (depending on $b$ ).

Definition 3.3. The spin rates $\{c(x, \eta)\}$ are said to have finite range if there exists $K \subseteq G$ finite such that if $\eta=\delta$ on $K$, then $c(e, \eta)=c(e, \delta)$.

We can assume that $e \in K$ and $K=K^{-1}$. In the above case, we say that $\{c(x, \eta)\}$ has finite range $K$ (although $K$ is not unique).

If $\left\{l_{1}, \ldots, l_{m}\right\} \subseteq G \times Z$, we let $\sigma\left(l_{1}, \ldots, l_{m}\right)$ denote the sub $\sigma$-field on $\{0,1\}^{G \times Z}$ generated by these $m$ points and let $\mathscr{A}\left(\sigma\left(l_{1}, \ldots, l_{m}\right)\right)$ denote the collection of $2^{m}$ atoms generating this sub $\sigma$-field. 
LEMMA 3.4. If the spin rates have finite range, then given any $b$ and $\xi>0$, one has that for all sufficiently invariant $F$, the following holds: If $\eta \in X$ and

$$
\left\{l_{1}, \ldots, l_{m}\right\} \subseteq(G \backslash F) \times[1, b],
$$

then for all $A \in \mathscr{A}\left(\sigma\left(l_{1}, \ldots, l_{m}\right)\right)$

$$
\bar{d}\left(\left\{Y_{i}^{\eta}(x)\right\}_{(x, i) \in F \times[1, b]},\left\{Y_{i}^{\eta}(x)\right\}_{(x, i) \in F \times[1, b]} / A\right) \leq \xi .
$$

Proof. Let $K$ be a finite set such that the spin rates have finite range $K$. By [1, Lemma 3.2], let $F$ be sufficiently invariant so that

$$
\frac{\left|F \backslash\left(\partial_{K^{2 b}} F\right)\right|}{|F|} \geq 1-\xi .
$$

Now, letting $S=F \backslash\left(\partial_{K^{2 b}} F\right)$, we have $K^{2 b} S \backslash F=\varnothing$, so $K^{b} S \cap$ $K^{b}(G \backslash F)=\varnothing$. Since the process has finite range $K$, it follows from the translation invariance that the process restricted to a set $A$ up to time $b$ only depends on the initial configuration on $K^{b} A$. Therefore for any $\eta \in X,\left\{Y_{i}^{\eta}(x)\right\}_{(x, i) \in S \times[1, b]}$ is independent of $\left\{Y_{i}^{\eta}(x)\right\}_{(x, i) \in(G \backslash F) \times[1, b]}$.

Therefore given $\left\{l_{1}, \ldots, l_{m}\right\} \subseteq(G \backslash F) \times[1, b]$, the way we have chosen $S$ implies that, for all $A \in \mathscr{A}\left(\sigma\left(l_{1}, \ldots, l_{m}\right)\right)$, the processes $\left\{Y_{i}^{\eta}(x)\right\}_{(x, i) \in S \times[1, b]}$ and $\left\{Y_{i}^{\eta}(x)\right\}_{(x, i) \in S \times[1, b]} / A$ have exactly the same joint distributions. Since

$$
\frac{|S \times[1, b]|}{|F \times[1, b]|} \geq 1-\xi
$$

this clearly implies that

$$
\bar{d}\left(\left\{Y_{i}^{\eta}(x)\right\}_{(x, i) \in F \times[1, b]},\left\{Y_{i}^{\eta}(x)\right\}_{(x, i) \in F \times[1, b]} / A\right) \leq \xi .
$$

THEOREM 3.5. If the spin rates are attractive and of finite range, then

$$
\left(\{0,1\}^{G \times Z}, \underline{\tilde{\nu}}\right)
$$

is Bernoulli.

Proof. This is proved along the same lines as Theorem 4.6 in [14], but we provide the proof. We demonstrate the Time Følner Independence property and then apply Theorem 2.5.2. Let $\varepsilon>0$. By Proposition 3.2, there is an integer $b$ such that, for all sufficiently invariant $F \subseteq G$, there is a set $E$ (depending on $F$ ) of $\underline{\nu}$-measure at most $\varepsilon$ such that

$$
\bar{d}\left(\left\{Y_{i}^{\eta}(x)\right\}_{(x, i) \in F \times[1, b]},\left\{Y_{i}(x)\right\}_{(x, i) \in F \times[1, b]}\right) \leq 8 \varepsilon^{1 / 4}
$$


for all $\eta \notin E$. By Lemma 3.4, for all sufficiently invariant $F$, for all $\eta$, if

$$
\left\{l_{1}, \ldots, l_{m}\right\} \subseteq(G \backslash F) \times[1, b],
$$

then for all $A \in \mathscr{A}\left(\sigma\left(l_{1}, \ldots, l_{m}\right)\right)$

$$
\bar{d}\left(\left\{Y_{i}^{\eta}(x)\right\}_{(x, i) \in F \times[1, b]},\left\{Y_{i}^{\eta}(x)\right\}_{(x, i) \in F \times[1, b]} / A\right) \leq \varepsilon .
$$

Together this gives us that for all sufficiently invariant $F$, there is a set $E$ of $\underline{\nu}$-measure at most $\varepsilon$ such that for all $\left\{l_{1}, \ldots, l_{m}\right\} \subseteq$ $(G \backslash F) \times[1, b]$,

$$
\bar{d}\left(\left\{Y_{i}^{\eta}(x)\right\}_{(x, i) \in F \times[1, b]} / A,\left\{Y_{i}(x)\right\}_{(x, i) \in F \times[1, b]}\right) \leq 9 \varepsilon^{1 / 4}
$$

for $\eta \notin E, A \in \mathscr{A}\left(\sigma\left(l_{1}, \ldots, l_{m}\right)\right)$. We claim that $F$ is $(N, \varepsilon)$-wide. Let

$$
\left\{y_{1}, \ldots, y_{r}\right\} \subseteq\left\{\omega \in G \times Z: \omega_{n+1} \leq b\right\} \backslash(F \times[1, b]) .
$$

Partition $\left\{y_{1}, \ldots, y_{r}\right\}$ into two sets $\left\{l_{1}, \ldots, l_{m}\right\}$ and $\left\{l_{1}^{\prime}, \ldots, l_{m^{\prime}}^{\prime}\right\}$, where the $l_{1}, \ldots, l_{m}$ all have their time coordinate positive and the $l_{1}^{\prime}, \ldots, l_{m^{\prime}}^{\prime}$ all have their time coordinate nonpositive. Then any element in $\mathscr{A}\left(\sigma\left(y_{1}, \ldots, y_{r}\right)\right)$ is $A_{1} \cap A_{2}$ where $A_{1} \in \mathscr{A}\left(\sigma\left(l_{1}, \ldots, l_{m}\right)\right)$ and $A_{2} \in \mathscr{A}\left(\sigma\left(l_{1}^{\prime}, \ldots, l_{m^{\prime}}^{\prime}\right)\right)$.

Now we let $Q^{A_{2}}$ denote the measure on $X$ obtained by considering the conditional distribution of $Y_{0}$ given $A_{2}$. By (3.1), if $\eta \notin E$, there is a measure $P^{\eta}, A_{1}$ on $\{0,1\}^{F \times[1, b]} \times\{0,1\}^{F \times[1, b]}$ which is a

$$
\left\{Y_{i}^{\eta}(x)\right\}_{(x, i) \in F \times[1, b]} / A_{1}-\left\{Y_{i}(x)\right\}_{(x, i) \in F \times[1, b]}
$$

coupling which gives $\bar{d}$-distance $\leq 9 \varepsilon^{1 / 4}$. If $\eta \in E$, we let $P^{\eta, A_{1}}$ be any

$$
\left\{Y_{i}^{\eta}(x)\right\}_{(x, i) \in F \times[1, b]} / A_{1}-\left\{Y_{i}(x)\right\}_{(x, i) \in F \times[1, b]}
$$

coupling. It then follows that

$$
P^{A_{1}, A_{2}}=\int_{X} P^{\eta, A_{1}} d Q^{A_{2}}(\eta)
$$

is a coupling of

$$
\left\{Y_{i}(x)\right\}_{(x, i) \in F \times[1, b]} /\left(A_{1} \cap A_{2}\right) \text { and }\left\{Y_{i}(x)\right\}_{(x, i) \in F \times[1, b]} .
$$

However, this is only a good coupling if $Q^{A_{2}}$ does not give too much measure to $E$. In particular, if $Q^{A_{2}}(E) \leq \sqrt{\varepsilon}$, then

$$
\begin{gathered}
\bar{d}_{P^{A_{1}, A_{2}}}\left(\left\{Y_{i}(x)\right\}_{(x, i) \in F \times[1, b]} /\left(A_{1} \cap A_{2}\right),\left\{Y_{i}(x)\right\}_{(x, i) \in F \times[1, b]}\right) \\
\leq 9 \varepsilon^{1 / 4}+\sqrt{\varepsilon}<10 \varepsilon^{1 / 4} .
\end{gathered}
$$


Since

$$
\sum_{A_{2} \in \mathscr{A}\left(\sigma\left(l_{1}^{\prime}, \ldots, l_{m^{\prime}}^{\prime}\right)\right)} Q^{A_{2}} \underline{\tilde{\nu}}\left(A_{2}\right)=\underline{\nu}
$$

and $\underline{\nu}(E) \leq \varepsilon$, it follows that

$$
\underline{\tilde{\nu}}\left\{\bigcup_{\left\{A_{2} \in \mathscr{A}\left(\sigma\left(l_{1}^{\prime}, \ldots, l_{m^{\prime}}^{\prime}\right)\right): Q^{A_{2}}(E) \geq \sqrt{\varepsilon}\right\}} A_{2}\right\} \leq \sqrt{\varepsilon} .
$$

Hence for all $A \in \mathscr{A}\left(\sigma\left(y_{1}, \ldots, y_{r}\right)\right)$ except for $\sqrt{\varepsilon}$ portion with respect to $\underline{\tilde{\nu}}$,

$$
\bar{d}\left(\left\{Y_{i}(x)\right\}_{(x, i) \in F \times[1, b]} / A,\left\{Y_{i}(x)\right\}_{(x, i) \in F \times[1, b]}\right)<10 \varepsilon^{1 / 4} .
$$

This, together with Lemma 2.1.1 demonstrates the Time Følner Independence property.

THEOREM 3.6. If the spin rates are attractive, then $\left(\{0,1\}^{G \times Z}, \underline{\tilde{\nu}}\right)$ is Bernoulli.

Proof. This follows easily from Theorem 3.5 and is proved exactly as is Theorem 4.7 in [14].

\section{REFERENCES}

[1] S. Adams, Følner Independence and the amenable Ising model, to appear, Ergodic Theory Dynamical Systems.

[2] _ - Very Weak Bernoulli for amenable groups, to appear, Israel J. Math.

[3] J. W. Kammeyer, A complete classification of the two-point extensions of a multidimensional Bernoulli shift, J. Analyse Math., 54 (1990), 113-163.

[4] J. C. Kieffer, A generalized Shannon-McMillan theorem for the action of an amenable group on a probability space, Ann. Probab., 3 (1975), 1031-1037.

[5] Thomas M. Liggett, Interacting Particle Systems, Springer-Verlag, 1985.

[6] Donald S. Ornstein, Ergodic Theory, Randomness and Dynamical Systems, Yale University Press, New Haven 1974.

[7] Donald S. Ornstein and B. Weiss, Entropy and isomorphism theorems for actions of amenable groups, J. Analyse Math., 48 (1987), 1-141.

[8] _ The Shannon-McMillan-Breiman theorem for a class of amenable groups, Israel J. Math., 44 (1983), 53-60.

[9] _ Every transformation is bilaterally deterministic, Israel J. Math., 24 (1975), 154-158.

[10] _ Finitely determined implies very weak Bernoulli, Israel J. Math., 17 (1974), 94-104.

[11] D. Rudolph, If a two-point extension of a Bernoulli shift has an ergodic square, then it is Bernoulli, Israel J. Math., 30 (1978), 159-180.

[12] P. Shields, Almost block independence, Z. Wahrscheinlichkeitstheorie Verb. Gebiete, 49 (1979), 119-123. 
[13] M. Smorodinsky, A partition of a Bernoulli shift which is not weakly Bernoulli, Math. Systems Theory, 5 (1971), 201-203.

[14] Jeffrey E. Steif, Space-Time Bernoullicity of the lower and upper stationary processes for attractive spin systems, Ann. Probab., 19, No. 2 (1991), 609-635.

[15] Peter Walters, An Introduction to Ergodic Theory, Springer-Verlag, New York 1975.

Received November 1, 1990 and in revised form, February 27, 1992. First author supported by NSF Postdoctoral Research Fellowship, Grant No. DMS 9007248. Second author partially supported by the Army Research Office through the Mathematical Sciences Institute at Cornell University and a National Science Foundation Mathematical Sciences Postdoctoral Research Fellowship.

Evans Hall

UNIVERSITY OF CALIFORNIA

BERKELEY, CA 94720

AND

White Hall

CORNELL UNIVERSITY

ITHACA, NY 14853 



\section{PACIFIC JOURNAL OF MATHEMATICS}

Founded by

E. F. BECKENBACH (1906-1982) F. Wolf (1904-1989)

\section{EDITORS}

\author{
V. S. VARADARAJAN \\ (Managing Editor) \\ University of California \\ Los Angeles, CA 90024-1555 \\ vsv@math.ucla.edu \\ F. Michael Christ \\ University of California \\ Los Angeles, CA 90024-1555 \\ christ@math.ucla.edu
}

\section{Herbert Clemens}

University of Utah

Salt Lake City, UT 84112

clemens@math.utah.edu

\author{
ThOMAs ENRIGHT \\ University of California, San Diego \\ La Jolla, CA 92093 \\ tenright@ucsd.edu \\ Nicholas Ercolani \\ University of Arizona \\ Tucson, AZ 85721 \\ ercolani@math.arizona.edu \\ R. FINN \\ Stanford University \\ Stanford, CA 94305 \\ finn@gauss.stanford.edu \\ VAUGHAN F. R. Jones \\ University of California \\ Berkeley, CA 94720 \\ vfr@math.berkeley.edu
}

STEVEN KeRCKHOFF

Stanford University

Stanford, CA 94305

spk@gauss.stanford.edu

Martin ScharlemanN

University of California

Santa Barbara, CA 93106

mgscharl@henri.ucsb.edu

Harold Stark

University of California, San Diego

La Jolla, CA 92093

\section{SUPPORTING INSTITUTIONS}

UNIVERSITY OF ARIZONA

UNIVERSITY OF BRITISH COLUMBIA

CALIFORNIA INSTITUTE OF TECHNOLOGY

UNIVERSITY OF CALIFORNIA

UNIVERSITY OF MONTANA

UNIVERSITY OF NEVADA, RENO

NEW MEXICO STATE UNIVERSITY

OREGON STATE UNIVERSITY
UNIVERSITY OF OREGON

UNIVERSITY OF SOUTHERN CALIFORNIA

STANFORD UNIVERSITY

UNIVERSITY OF HAWAII

UNIVERSITY OF UTAH

WASHINGTON STATE UNIVERSITY

UNIVERSITY OF WASHINGTON 


\section{PACIFIC JOURNAL OF MATHEMATICS}

Volume 159 No. $1 \quad$ May 1993

An application of the very weak Bernoulli condition for amenable groups

SCOT ROBERT ADAMS and JEFFREY EDWARD STEIF

An application of homogenization theory to harmonic analysis on solvable Lie groups of polynomial growth

G. Alexopoulos

The standard double soap bubble in $\mathbf{R}^{2}$ uniquely minimizes perimeter

Joel Foisy, Manuel Alfaro Garcia, JefFrey FARlowe

Brock, NiCKELOUS Hodges and JASON ZimbA

Pseudo regular elements and the auxiliary multiplication they induce

BARRY E. JOHNSON

A converse to a theorem of Komlós for convex subsets of $L_{1}$

CHRISTOPHER JOHN LENNARD

General Kac-Moody algebras and the Kazhdan-Lusztig conjecture

WAYNE L. NEIDHARDT

The flow space of a directed $G$-graph

WILLIAM LINDALL PASCHKE

Primitive ideals and derivations on noncommutative Banach algebras

MARK PHILLIP THOMAS

Equivariant Nielsen numbers

PETER N-S WONG

Volumes of tubular neighbourhoods of real algebraic varieties

RichaRd ALEXANDER WONGKEW

The intrinsic group of Majid's bicrossproduct Kac algebra 